\title{
Component-Based Automotive Production Systems
}

\author{
Richard H. Weston, Andrew A. West, and Robert Harrison \\ Loughborough University - MSI Res. Inst., r.h.weston@lboro.ac.uk
}

\begin{abstract}
The paper explains how 'process aware machine components' have been developed so that they can be reused as building blocks of 'in production' assembly and transfer machine elements used by a global consortium of companies making automotive products. It explains how computer executable models of components, alternative composition of machine components and responsive production lines provide common representations of production requirements and candidate solutions for reuse by members of distributed engineering teams. The findings are generalised in a philosophical discussion about 'change capable' systems and the current and future role of EM and EI in producing 'pro-active systems' capable of rapid 're-composition' and thence rapid and effective 'behavioural change'.
\end{abstract}

\section{PRODUCT AND PRODUCTION SYSTEM DECOMPOSITION IN SUPPORT OF GLOBAL MANUFACTURE}

The paper considers engineering processes used by a global consortium of businesses. A range of car engine types (known as I4/I5) was rationalised to facilitate mass customisation. Fig. 1 illustrates the product decomposition agreed by a number of car manufacturers who normally compete for business. For the new I4/15 engine product range these manufacturers will collaborate and use these product modules as standard components of different makes and models of car.

The I4/I5 product decomposition provides new opportunities to standardise and geographically distribute production and logistical activities involved in engine manufacture. The collaborating partners will enact these simplified 
production and logistical processes globally and thereby will supply I4/I5 engine variants in Europe, the Americas and Asia.

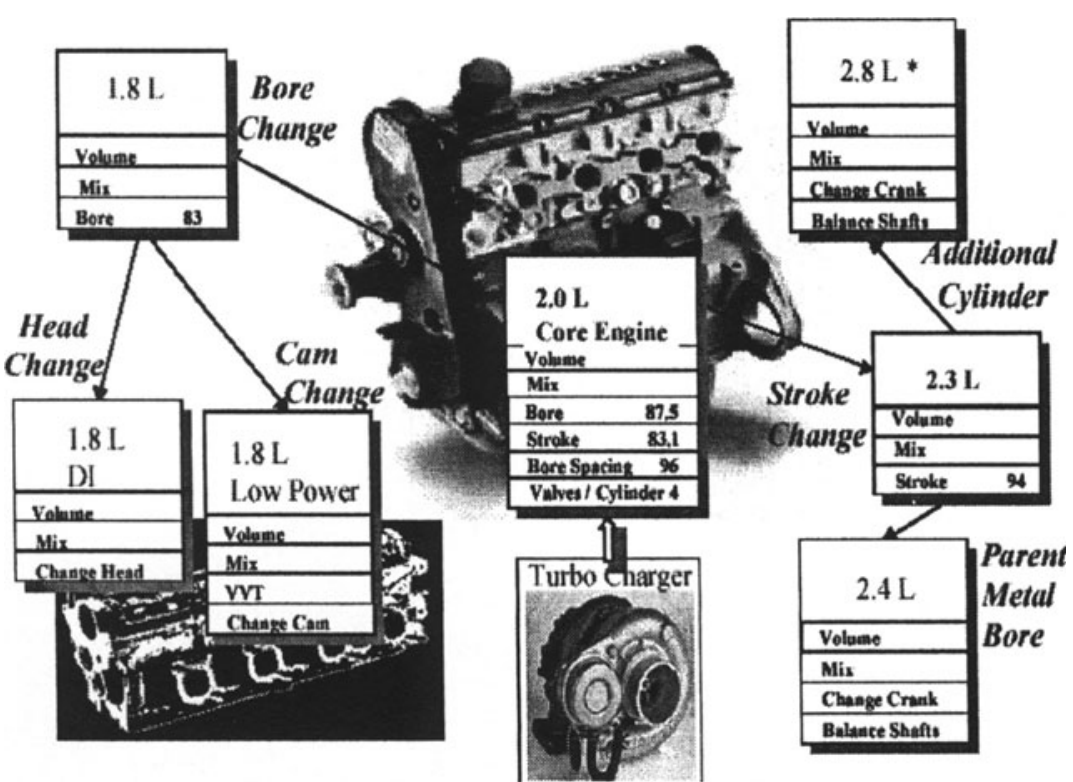

Figure 1: 14/15 Product Decomposition and Rationalisation Achieved by the Consor-

\subsection{Stage 1. Manufacturing System Decomposition}

To achieve standardisation of engine product design and to rationalise associated production processes, the collaborating car manufacturers have organised and managed the set of large-scale engineering projects (Fig. 2) (Harbers, 1996). It

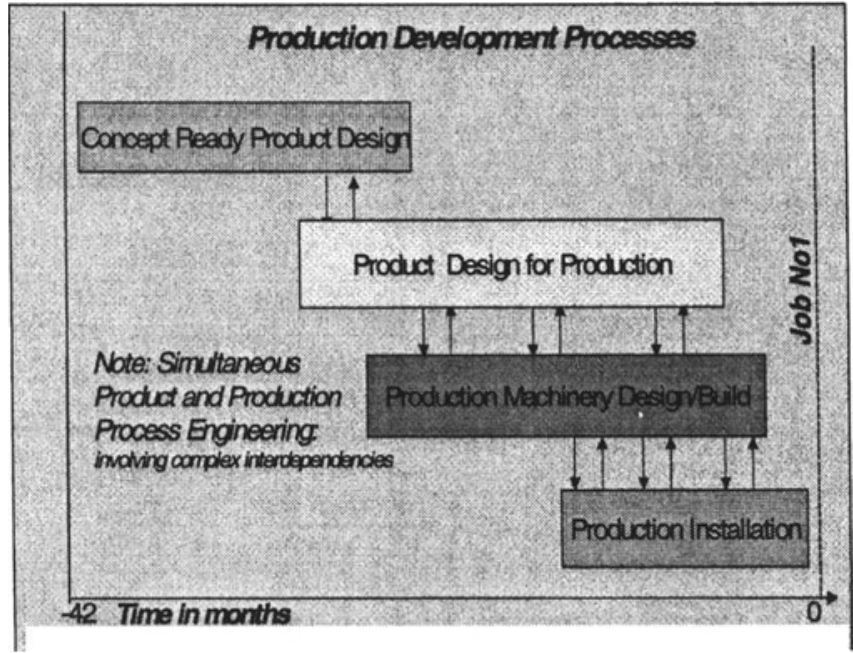

Figure 2: I4/I5 Product and Production Machinery Engineering Processes 
has been necessary for many activities involved in these projects to proceed in a concurrent manner, in order to compress lead-times. The I4/I5 engineering programme needed completing over a 42-month timeframe. At completion products will be sold to customers.

Naturally the engineering activities and processes involved are complex in their own right. Also activities and processes cannot wholly be decoupled from each other. Dependencies take a variety of forms typically involving cause and effect relationships that may change in space and time and have circular knock-on effects, one on another.

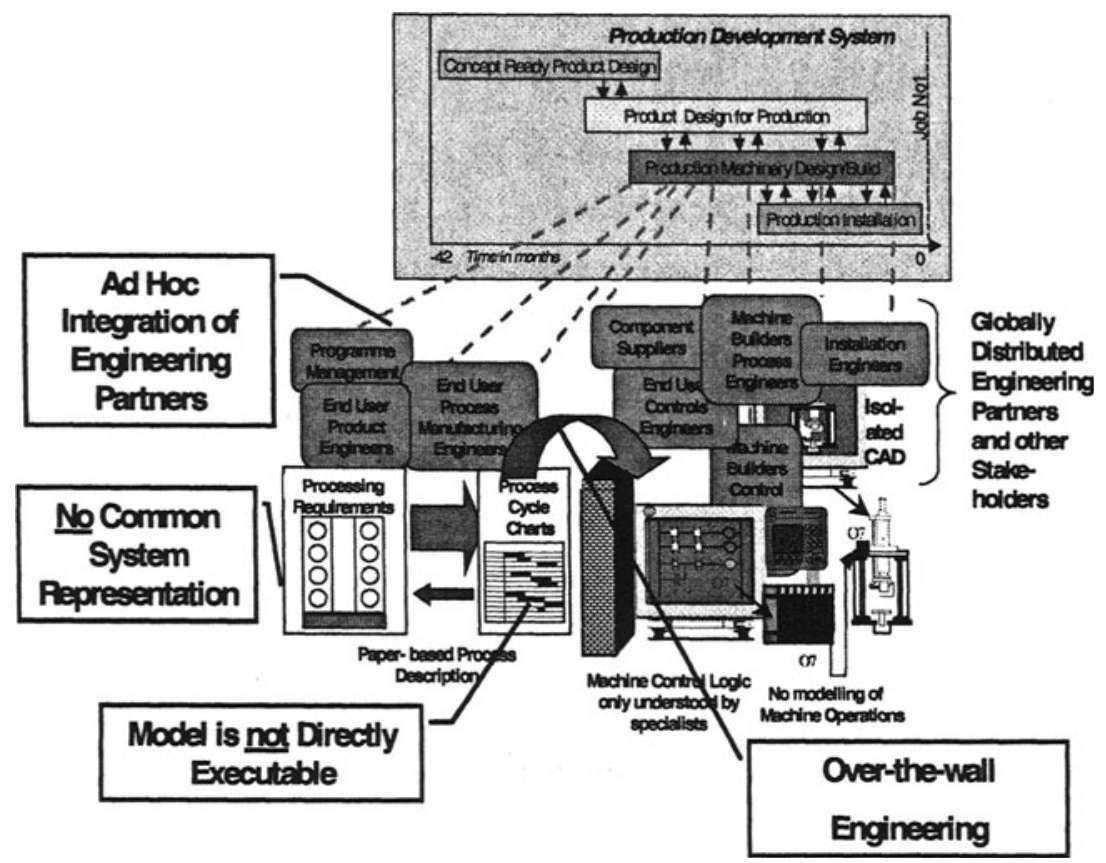

Figure 3: Current Production Machinery Design and Build Practice and Actors

The picture of dependency networks that link concurrent engineering processes to their products (which in this case are production and logistical processes needed to make automotive products) is further complicated because: (1) Necessarily engineering processes will be resourced by various teams of people, often with responsibilities aligned to different organisational units; (2) Engineering processes have a finite lifetime (up to 42 months in this case). Because of interdependencies making late but necessary change to the design and implementation of products, production processes (and associated production machine systems) can invalidate earlier engineering decisions and actions. This will result in re-engineering work and can impact significantly on costs and lead-times. I4/I5 production processes 
alone can involve over 5000 production line unit operations. Production facility development activities and flows are even more complex. Consequently change can propagate through and impact on a very large number of production engineering activities.

Essentially ad hoc integration methods and mechanisms are currently employed to support the people who enact 14/15 engineering activities. As illustrated in Fig. 3, machine and control system design and implementation is carried out by people who have various engineering roles and perform these roles using a fragmented set of specialist tools and methods that include computer aided design (CAD) tools, ladder logic programming tools and structured design and diagnostic and coding methods. The integration methods deployed to co-ordinate the use of these heterogeneous tools will at best be informally and uniquely specified (as best practice) in those companies responsible for production machine design and build. They do not utilise a common representation or visualisation of production machines throughout the lifecycle of machines, i.e. their design, analysis, implementation, testing, maintenance and reuse lifecycle. Nor is there any overall computer-executable model capable of supporting 'what if' analysis of alternative machine designs and behaviours (i.e. machine state changes over time). Consequently current production machinery design and build processes used in the automotive sector are not realised in a flexible way, in the sense that little support is provided for the reconfiguration and reuse of production machinery as products. The present engineering process used to design and build production machinery is also largely paper-based.

The automotive manufacturers and their partner machine builders are already aware that use of a suitable production system decomposition can help reduce the levels of complexity involved in: (a) engineering new products like I4/I5 and their production systems and (b) achieving and managing product and production facility change during engineering and production process lifetimes. However there are many possible 'entities' that could be 'modularised' and there will be multiple views (held for example by designers, builders, and managers within product manufacturer, machine builder and technology vendor partners of any global consortium) of what constitutes a 'good' production machine decomposition. Different decompositions can have conflicting business, technical and social implications. In practice any adopted decomposition will be a compromise, particularly because of uncertainty about future instances of change that might occur during the lifetime of products, production systems and production machines. Fundamental reasons to compromise will also arise because some entities cannot be broken down effectively, possibly because of natural couplings to other entities. The machine builder will have concerns about what will be acceptable to other customers during the lifetime of machine system modules (viewed as a 
product) and other uncertainties caused by a changing base of enabling methods and techniques. While the product manufacture will be concerned primarily about the implications of product change and/or change in production methods requiring new product mixes to be realised.

Consequent on these factors machine builder partners of the I4/I5 consortium have continued cautiously but progressively to modularise their relevant machinery by producing mechanical and control system elements that can readily be configured and programmed so that they meet specified user (manufacturer) production requirements adequately well. This has helped end user car manufacturers to standardise production processes and machine builders to design, make and reuse 'modules' of production machines.

End user manufacturers have continued to rationalise the engineering and production processes they deploy. Typically their processes are now described in terms of well defined units of 'operation', 'activity' or 'task'. The automotive industry at large has also understood a need to select and deploy suitable types of team (comprising people and technology) to resource production and engineering activities. However like their machine builders generally end user manufacturers have rationalised and standardised their processes and systems asynchronously from individual new product developments. Normally rationalisation, simplification, modularization and standardisation have come from understanding best custom and practice over a number of product iterations and represent a compromise solution that is acceptable to key parties over the lifetime of a number of products. This state of affairs can be seen as a natural one. However, the current outcome is far from optimum in the face of requirements for reduced product lifetimes, increased product variety and the need for multiparty consortia to come together in partnership to satisfy a specific global product and service requirement. A concrete but simple illustration of the deficiency of the status quo is illustrated on considering the projected relative lifetimes of I4/I5 engines and I4/I5 production lines, where the latter is likely to be around twice that of the former. The implication is that either over its lifetime 'production line utilisation' will be low or that production lines must be reconfigured around halfway through their lifetime to enable their reuse when producing the next product generation. It is observed that the current engine assembly production machine decomposition has ignored at least one important view, i.e. the view that automotive production machines need to be reused for more than one product and that embedding of a suitable change capability into a new generation of production machines might save tens of million US dollars during the lifetime of a single production line. 


\section{RESEARCH STEPS TAKEN TOWARDS REALISING A NEW GENERATION OF COMPONENT-BASED PRODUCTION MACHINES}

Early in the I4/I5 product and process engineering programme the partner companies recognised that significant potential business benefit could accrue from realising an improvement in the 'change capability' of production machines. It was understood that advances in distributed computing component technologies and enterprise modelling might be harnessed to facilitate: (a) Faster and significantly lower cost first-off machine design and build; (b) Significantly faster and lower cost mid-life machine 'recomposition' and 'reconfiguration'; (c) More effective reuse by machine builders and their technology suppliers of machine modules at various end-user sites.

A programme of research was funded by the partner companies and the UK research councils. The collective aim was to prototype and industrially test a new generation of 'change capable' engine assembly production systems. Much of the research work has been carried out at Loughborough University, in parallel with the conventional I4/I5 product and production system engineering work of the industrial partners. Research assumptions being tested are that:

a) A suitable 'to be' decomposition of automotive production systems into mechatronic components can be identified, practically realised and supported on an industry-wide scale. Machine components need to be reused in 'composable production systems' that provide plant specific capabilities needed to assemble customer specified quantities of car engine types.

b) Key properties of production operations, mechatronic components and various production machine systems composed from the components, can be adequately modelled. The purpose being to match models of composed machines to models of production needs (i.e. production activities and processes) within a virtual environment for machine engineering.

c) Engineering activities needed to design and build component-based automotive production systems can be represented and simulated with sufficient realism to facilitate improved engineering process design and resourcing by engineering teams. By modelling engineering activities the aim was to predict benefits arising from using standard production machine components and to facilitate the design of a virtual environment (and supporting infrastructure services) with capa- 
bility to support and co-ordinate the distributed interworking of teams of engineers.

d) A suitable and effective set of engineering tools specified under (3) can be prototyped and their use demonstrated and appraised by consortium partners.

SAMPLE OF RESEARCH RESULTS

CIMOSA modelling concepts were deployed to structure the capture of multi-perspective views of 'as is' practice used by I4/I5 engineering partners as they define production and logical processes and design and make car engine assembly plants at various sites around the globe. The approach allowed multiple views of current practice to be captured in a standardised and coherent fashion. The data captured has been used in numerous ways by MSI researchers. For example, iThink dynamic system models have been generated using this data that simulate the distributed operation of machine engineering processes amongst partner companies. Also workflow models of engineering activities in machine builder companies have been generated using the iFlow tool with a view to achieving improved engineering management and control. The modelling studies have contributed towards the development and documentation of new multi-perspective understandings about engineering processes, structures, resources and services needed to realise car assembly plants. Ongoing research is developing the use of this multi-perspective knowledge with a view to specifying a semi-generic (domain) architecture for component-based engine assembly machines.

The research has also specified and developed an exemplar set of engine assembly 'machine components' that conform to the multi-perspective domain architecture. Component design has been achieved in two complementary ways, namely:

a) By achieving a next step elemental decomposition, of existing mechanical modules produced by machine builder partners, and embedding a suitable control system decomposition into mechanical elements based on using Fieldbus and distributed object technologies.

b) By embedding common runtime models into mechatronic and virtual components so that (i) individual component behaviours and interlock and exception conditions can be programmed, controlled and monitored and (ii) the collective behaviours and conditions of composed groupings of components can be matched to production process needs and controlled and monitored in conformance with the multiperspective domain architecture. 
Another main focus of research has been on specifying, developing and appraising the industrial use of an exemplar distributed engineering environment (Harrison, 2001) Fig. 4 illustrates some of the tools and concepts embedded into this environment. An initial set of engineering tools and infrastructure services has been prototyped. A base level of support is provided for distributed teams of interdisciplinary engineers and managers (from machine customers, builders and technology providers with responsibilities for engineering, operating, maintaining and changing production machines.

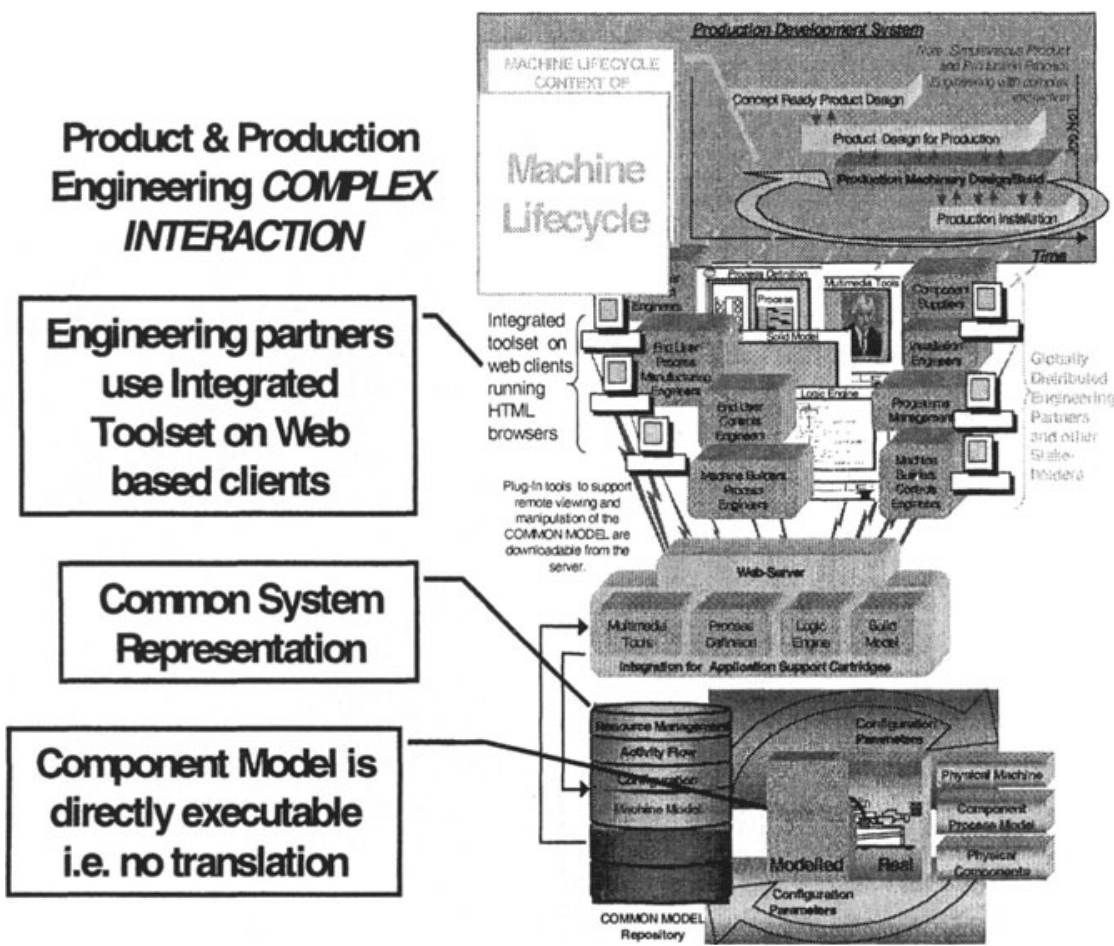

Figure 4: Characteristics of the Prototype Distributed Engineering Environment

Fig. 5 shows in concept how it has proven practical and effective to maintain consistency and coherence between runtime behaviours and exception conditions encoded by models of production processes and individual machine component behaviours by using engineering tools that collectively form a distributed (virtual) engineering environment. By such means many views of components and composed systems can be created and manipulated by suitable software tools located at their point of use. For example, models of machine system kinematics (in the virtual environment) can be 'connected' to actual machine behaviours and feedback data. Thereby it has proven possible to create multiple visualisations of virtual and real machine behaviours. The multi-perspective domain model provides a contextual 
framework that connects the generation and use of these modelling views to engineering actors as they carry out engineering activities pertaining to the lifecycle of the machine.

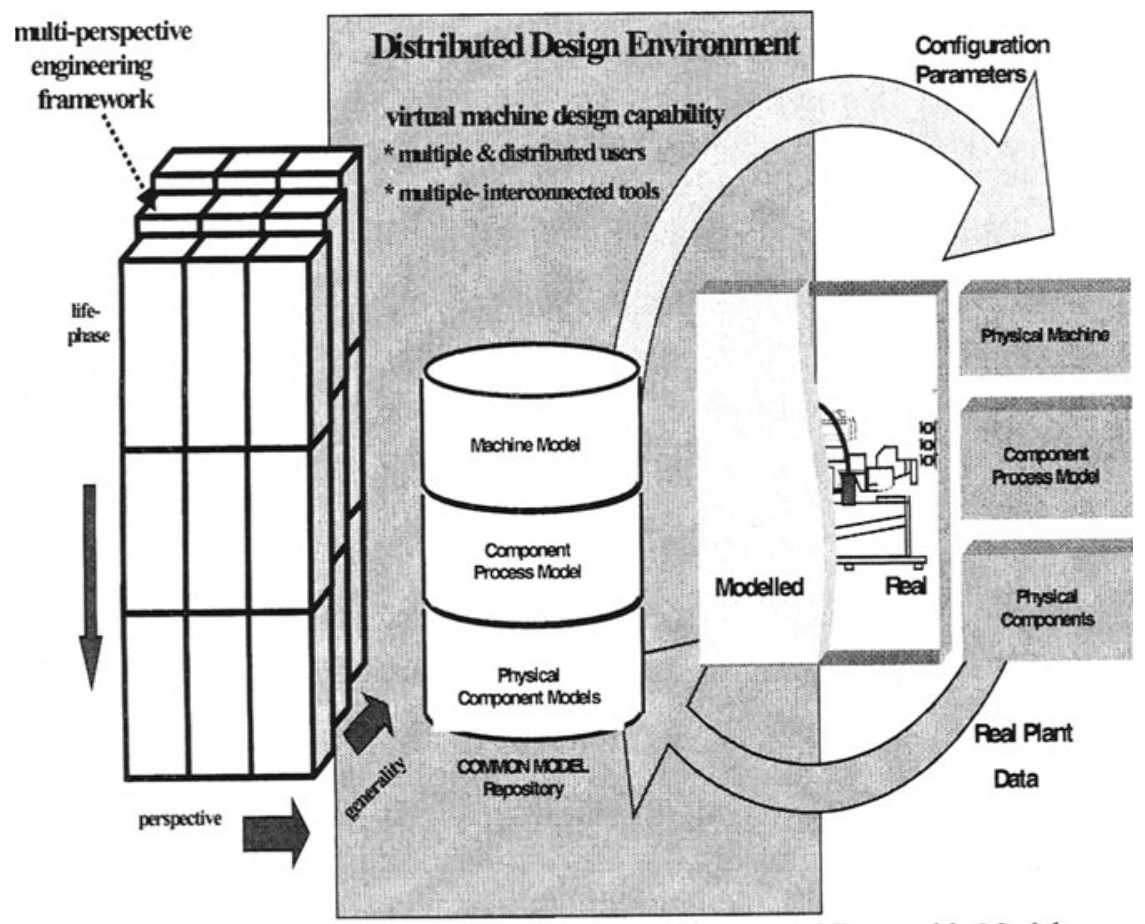

Figure 5: Example Mechatronic Component and its Integral Executable Models

Prototype testing and ongoing development of the new component-based approach to engineering engine assembly machines is ongoing in MSI laboratories. In the first quarter of 2002 prototype-testing work had begun at the promises of two machine builder partners. The concepts and their first generation implementation show significant promise from both practical and theoretical perspectives.

\section{SOME GENERAL OBSERVATIONS AND LESSONS LEARNT}

As a consequence of moving to the 'to be' component-based engineering approach it is anticipated that significant cost savings would result from new I4/I5 plant installations around the globe and in other domain systems. For example, a $50 \%$ savings in commissioning time is expected with typical cost benefits equating to circa US\$20M. Improved machine operation, mainte- 
nance and minor change will potentially save US\$50M during the machine lifetime through halving downtimes. Also 'mid life' product change will save between US\$6M and US\$15M. However, significant research and development resource has been expended in developing the new approach (order of US\$2M). Much greater product development resource will be needed to develop the approach into an industry-wide technology that can be supported in the field but although some of the concepts used in developing the multi-perspective domain architecture, the machine components and the virtual environment can be reused in other engineering domains, generally any new domain application of the concepts will be distinct and require the capture of new multiparty domain knowledge. Use of project methods, many of which are based on public domain concepts like CMIOSA concepts can facilitate this process. But generally current generation enterprise modelling tools have proven deficient in their lifecycle engineering scope, coverage of modelling views and ease of integration with other tools, and this will limit reuse of the project methods.

The research pointed up new areas where enterprise modelling, engineering and integration research is needed. One key area of unsatisfied need is that of managing dependencies between different aspects of engineering and production processes. Although commercial enterprise modelling tools, data management and versioning tools and advance of transaction processing tools can be deployed generally they only address part of this problem. A coherent rather than fragmented technology is needed so that various types of change can be managed effectively. New research is needed to address inadequate interprocess co-ordination. Two co-ordination concerns could not be effectively satisfied, namely: (1) where a process with finite lifetime is executing and essentially dynamic (or runtime) change is needed. Typically solution to such problems requires rich process descriptions and improved commercial modelling tools with capability to capture and reuse state data (present and past) within change processes; (2) Where process descriptions are distributed into autonomously executing components but should operate coherently (but in a change capable way) with respect to a component group and/or backbone process execution.

\section{REFERENCES}

Harbers, W. O., (1996), Ford Automation Strategies and Needs, Automation Research Corporation: Automation Strategies Forum, Boston, Massachusetts, June.

Harrison, R, West, A.A., Weston, R.H. Monfared, R.P. (2001), Distributed Engineering of Manufacturing Machines, Journal of Eng. Manuf., Part B2, Vol. 215, pp 217-231, March. 